NBER WORKING PAPER SERIES

ESTIMATING THE LONG-RUN RELATIONSHIP BETWEEN INTEREST RATES AND INFLATION:

A RESPONSE TO MCCALLUM

Lawrence H. Summers

Working Paper No. 1448

\author{
NATIONAL BUREAU OF ECONOMIC RESEARCH \\ 1050 Massachusetts Avenue \\ Cambridge, MA 02138 \\ September 1984
}

I am indebted to Robert Barsky for research assistance and helpful discussions, to Brad DeLong for helpful discussions and to the NSF for financial support. The research reported here is part of the NBER's research programs in Economic Fluctuations and Financial Markets and Monetary Economics. Any opinions expressed are those of the author and not those of the National Bureau of Economic Research. 
NBER Working Paper \#1448 September 1984

\author{
Estimating the Long-Run Relationship Between \\ Interest Rates and Inflation: \\ A Response to McCallum
}

\begin{abstract}
This note demonstrates that Bennett McCallum's recent critique of low frequency estimates of macro-economic relationships is of little empirical significance. It also demonstrates that readily available and frequently used techniques can be used to diagnose the problem McCallum raises. Finally, it shows that the standard critique of expectational distributed lags is not warranted once the role of learning by economic agents is recognized.
\end{abstract}

\author{
Lawrence H. Summers \\ Department of Economics \\ Littauer Center 229 \\ Harvard University \\ Cambridge, MA 02138
}


In a recent contribution to this Journal Bennett McCallum (1984) sharply criticizes econometric tests of long run economic relationships, which make use of frequency domain time series techniques. Summers (1982) is held out as an offending example. This note demonstrates that McCallum's criticisms are not valid either theoretically or empirically. It makes three points. First, as asserted in original paper, the issue of measuring inflation expectations, which is the heart of McCallum's critique, is of limited empirical significance in low frequency studies of the Fisher effect. Second, very simple econometric procedures implemented in original paper and in another paper criticized by McCallum make it possible to bound the bias arising from the problems McCallum raises. The potential bias is small. Third, once the role of learning is recognized, the Sargent-Lucus critique of expectational distributed lags which provides the basis for McCallum's comment is incorrect.

\section{Measuring Expected Inflation}

McCallum summarizes his comment by noting that "the low frequency measures in question are simply not designed to reflect the distinction between anticipated and unanticipated fluctuations that is crucial for accurately characterizing inter variable relationships in dynamic models." The central issue of measuring inflation expectations was recognized in my original paper. Indeed the Appendix contains an exposition of Sargent's (1971) point which underlies McCallum's comment. The text also addresses the issue of measuring inflationary expectations and makes the empirical assertion that "low frequency variations in the rate of inflation are almost completely forecastable so that the assumption that expected inflation can be proxied by actual inflation is warranted. 
Indeed, when the equations reported here were re-estimated with various proxies for expected inflation the results were not significantly affected."

Below this asssertion is documented using measures of expected inflation which are free from the Lucas-Sargent-McCallum taint. Before turning to this empirical evidence it should be made clear that the motivation for performing low frequency econometric tests is the conviction that history should not be viewed as a sequence of realizations of a single stationary stochastic process. The data can only illuminate long-run issues if regime changes of the type envisaged in comparative steady state economic theory have actually taken place. The possibility that such changes may take place and that if they do low frequency econometric techniques may provide a good way to study their effects is not disputed by McCallum. If regime changes have taken place, they will acccount for most of the variance in both expected and actual inflation, so that actual inflation will, at low frequencies, be a satisfactory proxy for expected inflation.

Ultimately then, the validity of the low frequency econometric techniques used in my paper is an empirical question depending on the properties of the inflation process. I tried to resolve it in preliminary work on the paper by seeing if substantively different empirical results regarding the Fisher effect were obtained when proxies for expected inflation were used. A rolling ARMA procedure of the type first used in Feldstein and Summers (1978) was employed. In this procedure each period a measure of expected inflation is $\Pi^{\mathrm{e}}$ by forecasting inflation based on an ARMA estimated over the previously available data. This method produces direct estimates of $\pi^{e}$ which depend on the 
stochastic process actually followed by inflation, and so is free of the problems raised by McCallum. Note that McCallum raises no objection to the low frequency estimation of a structural relation between interest rates and a direct measure of inflationary expectations. His only difficulties involve the use of distributed lags in measuring inflationary expectations.

Some of the results in original paper are reproduced along with results using proxies for expected inflation in Tables 1 and 2 . The alternative results suggest larger estimates of the effect of inflation on interest rates than do those in earlier paper. However, its principal conclusions are confirmed. For the 1860-1940 period, the hypothesis that the Fisher coefficient is one is rejected. For the Post-War interval, the hypothesis that the Fisher coefficient equals its tax adjusted value is also rejected. However, in some sub-samples, the use of the alternative inflation variables does have dramatic effects. This is particularly the case for the 1954-1971 interval, where the Fisher coefficient rises from .79 to 1.39 , at a frequency of over 3 years. The observation that over short intervals the choice of an expectations measure has significant effects should not be too surprising since they are less likely to contain regime changes of the type discussed above.

\section{Bounding Possible Errors ${ }^{1}$}

The analysis so far establishes the empirical irrelevance of McCallum's criticism of evidence pointing to the non-adjustment of nominal interest rates. This section reconsiders McCallum's econometric argument and the next section reexamines its theoretical basis.

Consider McCallum's example: 


$$
\begin{aligned}
& i_{t}=\rho+E_{t} \Pi_{t+1}+v_{t} \\
& \Pi_{t}=\mu_{0}+\mu_{1} \Pi_{t-1}+e_{t}
\end{aligned}
$$

where I have changed his notation but nothing else. McCallum's analysis focuses on low frequency estimates of the relationship:

$$
1_{t}=\left(\rho+\mu_{0}\right)+\mu_{1} \Pi_{t}+v_{t}
$$

He notes that a researcher estimating this relationship in either the time or frequency domain would conclude that the Fisher relationship failed to hold even though it was built into the econom by assumption in (1) as long as $\mu_{1}<1$. Equation (3) is temporally misaligned relative to the actual estimation presented in Summers (1982),

$$
i_{t}=\left(\rho+\mu_{0}\right)+\beta \pi_{t+1}+u_{t}
$$

where the actual realization of inflation is used as a proxy for its expectation. 2 McCallum in a footnote asserts that the distinction between (3) and (4) is immaterial. To see that this is in general incorrect, consider the special case where $e_{t+l}$ is perfectly forcastable using information available to agents but not available to econometricians. Then clearly, $\Pi_{t+1}=E\left(\Pi_{t+1}\right)$ and so (4) provides a legitimate test for the Fisher effect even though (3) does not.

As McCallum (1976) himself was the first to point out, (4) can be thought of as having the classical errors in variables problem. Under the assumption of rational expectations, $\pi_{t+1}$ is a noisy measure of $E_{t}\left(\pi_{t+1}\right)$. This means that $B$ will be biased towards 0 . As Delong (1983) demonstrates, a parallel result to be classical errors in variables formula holds in the frequency domain as well. 
There is no deep identification problem here, only one of errors in variables.

A primitive solution to the errors in variables problem is to bound the bias by running the regression equation in the opposite direction. In fact this is not necessary in the two variable case since the reverse regression coefficient can be calculated as the ratio of the $R^{2}$ to the slope coefficient in the original regression. This primitive solution to the errors in variables (and simultaneity) problem was discussed and implemented in original paper. Implicitly, it was also implemented by Lucas (1980) who exhibits his data graphically without explicitly estimating regressions. The results in my work suggested that in almost all cases, the point estimates bounded the effect of expected inflation on interest rates below that predicted by the Fisher theory. An alternative procedure for calculating the possible bias in band spectral estimates of (4) is to calculate explicitly the noise to signal ratio for $\mathrm{Il}_{t}$ as a measure of $E\left(\pi_{t}\right)$. This approach is taken by Delong (1983) who concludes that, "there is no Fisher effect before World War II and after World War II it is not possible to believe both in the distortionary effects of the tax system and the Fisher effect."

There is both a methodological and a substantive point in this section. The latter confirms in a different way the empirical analysis of the first section suggesting that problems of measuring inflation expectations are not an important explanation for negative results regarding the Fisher effect. The methodological point is that it is easy to determine whether expectational errors are an important problem, and to estimate the magnitude of any biases they engender. 


\section{The Validity of Expectational Distributed Lags}

As the previous section made clear, the use of actual inflation as a proxy for expected inflation involves errors in variables problems, but not deeper issues of the type raised first by Sargent (1971). Here, I demonstrate that even if lagged rather than actual values of inflation had been used in the analysis, there would be no serious problem. The now traditional critique of identification in distributed lag models is incorrect once the need for economic agents to learn the true economic model is recognized. The point made here is spelled out in more detail, and its implications for rational expectations econometrics are spelled out in more detail in Summers (1984).

Consider again McCallum's example given in (1) and (2). McCallum and Sargent (1971), (1973) implicitly argue that a regression of $i_{t}$ on any distributed lag of past $\pi_{t}$ would yield a coefficient of $\mu_{1}$ on $\pi_{t}$ and 0 on all lagged Is. This argument implicitly assumes that agents know $\mu_{0}$ and $\mu_{1}$ divinely and need not try to infer them from the data, unlike underprivileged econometricians. Friedman (1980) discusses the implausibility of this assumption.

Assume on the other hand that agents always use some finite amount of past data to estimate the parameter $\mu_{0}$ in (2).3 If their estimates are unbiased, they mast have the property that:

$$
\rho_{0}+\mu_{1} \bar{\Pi}=\bar{\Pi}
$$

or equivalently that:

$$
0_{0}=\left(1-\mu_{1}\right) \bar{\Pi}
$$

where $\bar{\Pi}$ refers to the mean value of $\pi$ in the sample period over which the para- 
meter $p_{0}$ is being estimated. Suppose for example that agents always use only the most recent $N$ observations in estimating $D_{0}$. Then an estimate of (3) in which additional lagged values were included would yield:

$$
i_{t}=p+\mu_{1} \Pi_{t}+\left(1-\mu_{1}\right) \sum_{i=0}^{N-1} \frac{1}{N} \Pi_{t-i}+e_{t}
$$

Note that the weights on the inflation terms in (7) sum to unity. This result generalizes easily to the case where $\Pi_{t}$ follows a more complicated process than (2). It also can be generalized to allow for alternative learning procedures in which the weight given to past data declines gradually.

A more significant generalization of this result proceeds as follows. Consider any method by which agents try to discern structure and forecast a stationary time series. Estimation and forecasting using time series models is one example of such a method. Any explicitly described method will give rise to a functional relationship between forecasts and past data of the form: ${ }^{4}$

$$
\Pi_{t+1}=F\left(\Pi_{t}, \Pi_{t-1}, \ldots . \Pi_{t-N-1}\right)
$$

This function can be approximated by using its Taylor expansion about the sample mean value of $\Pi$. This yields:

$$
\Pi_{t+1}=F\left(\pi_{t} \Pi \ldots \pi_{t-N-1}\right)+\sum_{i=0}^{N-1} \gamma_{i}\left[\Pi_{t-i}-\pi\right]
$$

where $\gamma_{i}=\left(\frac{\partial F}{\partial I I_{t-i}}\right) \bar{\Pi}_{\bar{\Pi}}$. If we impose the minimal rationality requirement on the function $F$ that $F\left(\pi, \pi \ldots \pi_{N-1}\right)=\bar{\pi}$, we obtain:

$$
\Pi_{t+1}=\bar{\Pi}+\sum_{i=0}^{N-1} \gamma_{i} \Pi_{t-i}-\bar{\Pi} \sum_{i=0}^{N-1} \gamma_{i}
$$


Substituting for $\bar{\Pi}$ using the definition $\bar{\Pi}=\frac{1}{N} \sum_{i=0}^{N-1} \Pi_{t-i}$ yields

$$
\pi_{t+1}=\sum_{i=0}^{N-1}\left[\frac{1}{N}+r_{1}-\frac{1}{N} \Gamma\right] \pi_{t-i}
$$

where $\Gamma=\sum_{i=0}^{N} \gamma_{i}$. The sum of the bracketed terms in (10) is unity. This establishes that in general learning procedures applied to finite bodies of data which satisfy a minimal rationality requirement will have the property that they are best approximated as weighted averages of past data with weights sumning to unity. 5

Thus, once agents need to learn the mean inflation rate is recognized, traditional expectational distributed lags and their maintained hypothesis that the sum of the lag weights used in forming inflation expectations is unity are vindicated. McCallum's equation (9) shows that when the sum of the weights on lagged inflation used in forecasting inflation is unity, band spectral regressions will provide a legitimate test of the Fisher effect. Of course, depending upon how agents process information, the lag length may be quite long. But this corresponds to the available empirical evidence on inflation and interest rates, particularly for the 1860-1940 period, and also confirms the desirability of using low frequency techniques.

\section{Conclusions}

The point McCallum makes while technically correct is of negligible substantive importance for studies of the Fisher effect. Nor should his paper 
$$
-9-
$$

deter future investigations from using low frequency econometric techniques in appropriate settings. There is no econometric "wonder technique" that will give right answers in all settings. Empirical workers have at their disposal a variety of techniques which will work well in some situations and poorly in others. Successful empirical work requires an appropriate match between statistical technique and the data and question being examined. In order to be telling, criticism of econometric techniques mast go far beyond demonstrating that conceivable settings exist where they will not yield meaningful results. While McCallum shows that such settings exist for band spectral techniques, this does not call into questions their utility in the setting where they have been applied. If economists are going to test the "long run" implications of their theories, some technique like band spectral regression will be necessary.
\end{abstract}




\section{Footnotes}

1. This section draws heavily on DeLong (1983).

2. Because of problems of data alignment, the equation estimated may not be exactly equivalent to (4).

3. We assume implausibly that $\mu_{1}$ is known. This assumption is relaxed below.

4. One example of a learning procedure would be the rolling ARMA method described in Section $I$. By making $N$ arbitrarily large, any procedure can be approximated. Note that there is no requirement that observations be used symmetrically. Giving more weight to recent data is consistent with (7).

5. Note that it is possible that some of the $\gamma_{i}$ will be negative. This will occur if plausible learning procedures are applied to non-stationary series. 


\section{$\underline{\text { References }}$}

DeLong, B., "Observations on the Non-Adjustment of Nominal Interest Rates," Mimeo (1983).

Engle, Robert F., "Band Spectrum Regression," International Economic Review 15 (February 1974), 1-11.

Feldstein, Martin, and Lawrence Summers, "Inflation Tax Rules, and the Long Term Interest Rate," BPEA 1 (1978), 61-109.

Friedman, Benfamin M., "Rational Expectations and the Extreme Information Assumptions of Rational Expectations Macromodels," Journal of Monetary Economics, 1979 .

Lucas, Robert E., Jr., "Econometric Testing of the Natural Rate Hypothesis," in 0. Eckstein, ed., The Econometrics of Price Determination Conference (Washington: Board of Governors of the Federal Reserve System, 1972) (a). , "Two Illustrations of the Quantity Theory of Money," American

Economic Review 70 (December 1980), 1005-1014.

McCallum, Bennett, "Rational Expectations and the Natural Rate Hypothesis," Econometrica (1976), 43-52. , "On Low Frequency Estimates of "Long Run" Relationships in Macroeconomics," Journal of Monetary Economics, June 1984. Sargent, Thomas J., "A Note on the Accelerationist Controversy," Journal of Money, Credit, and Banking 3 (August 1971), 50-60. , "Rational Expectations, the Real Rate of Interest, and the Natural Rate of Unemployment," Brookings Papers on Economic Activity (No. 2, 1973) 429-372. 
, Macroeconomic Theory (New York: Academic Press, 1979).

Summers, Lawrence H., "The Non-Adjustment of Nominal Interest Rates: A Study of the Fisher Effect," in J. Tobin, ed., Essays in Honor of Arthur Okun (1982).

, "Rational Learning Mechanisms Implying Irrational Expectations," in preparation, 1984 . 